PROCEEDINGS OF THE

AMERICAN MATHEMATICAL SOCIETY

Volume 128, Number 8, Pages 2329-2335

S 0002-9939(99)05273-9

Article electronically published on December 7, 1999

\title{
CARLESON MEASURES AND SOME CLASSES OF MEROMORPHIC FUNCTIONS
}

\author{
RAUNO AULASKARI, HASI WULAN, AND RUHAN ZHAO
}

(Communicated by Albert Baernstein II)

\begin{abstract}
For $|a|<1$ let $\varphi_{a}$ be the Möbius transformation defined by $\varphi_{a}(z)=\frac{a-z}{1-\bar{a} z}$, and let $g(z, a)=\log \left|\frac{1-\bar{a} z}{z-a}\right|$ be the Green's function of the unit disk $\mathcal{D}$. We construct an analytic function $f$ belonging to $M_{p}^{\#}=\{f$ : $f$ meromorphic in $\mathcal{D}$ and $\left.\sup _{a \in \mathcal{D}} \iint_{\mathcal{D}}\left(f^{\#}(z)\right)^{2}\left(1-\left|\varphi_{a}(z)\right|^{2}\right)^{p} d A(z)<\infty\right\}$ for all $p, 0<p<\infty$, but not belonging to $Q_{p}^{\#}=\{f: f$ meromorphic in $\mathcal{D}$ and $\left.\sup _{a \in \mathcal{D}} \iint_{\mathcal{D}}\left(f^{\#}(z)\right)^{2}(g(z, a))^{p} d A(z)<\infty\right\}$ for any $p, 0<p<\infty$. This gives a clear difference as compared to the analytic case where the corresponding function spaces $\left(M_{p}\right.$ and $\left.Q_{p}\right)$ are same.
\end{abstract}

Let $\mathcal{D}=\{z \in \mathbb{C}:|z|<1\}$ be the unit disk, and denote by $\partial \mathcal{D}$ the boundary of $\mathcal{D}$. For $a \in \mathcal{D}$, let the Möbius transformation $\varphi_{a}: \mathcal{D} \rightarrow \mathcal{D}$ be defined by

$$
\varphi_{a}(z)=\frac{a-z}{1-\bar{a} z}, \quad z \in \mathcal{D} .
$$

For $0<r<1$, let $\mathcal{D}(a, r)=\left\{z \in \mathcal{D}:\left|\varphi_{a}(z)\right|<r\right\}$ be the pseudohyperbolic disk with center $a$ and radius $r$, and let $g(z, a)=\log \left|\frac{1-\bar{a} z}{z-a}\right|$ be the Green's function on $\mathcal{D}$ with logarithmic singularity at $a \in \mathcal{D}$.

For a subarc $I \subset \partial \mathcal{D}$, let

$$
S(I)=\{z \in \mathcal{D}: 1-|I|<|z|<1, z /|z| \in I\} .
$$

If $|I| \geq 1$, then we set $S(I)=\mathcal{D}$. For $0<p<\infty$, we say that a measure $\mu$ defined on $\mathcal{D}$ is a bounded $p$-Carleson measure if

$$
\sup \left\{\mu(S(I)) /|I|^{p}: I \subset \partial \mathcal{D}\right\}<\infty .
$$

If $p=1$, we get the classical Carleson measure (see [8, p. 238]).

For $0<p<\infty$, the authors in [1] and [4] considered the space $Q_{p}$ as the following:

$$
Q_{p}=\left\{f: f \text { analytic in } \mathcal{D} \text { and } \sup _{a \in \mathcal{D}} \iint_{\mathcal{D}}\left|f^{\prime}(z)\right|^{2}(g(z, a))^{p} d A(z)<\infty\right\},
$$

where $d A(z)$ is the usual element of Euclidean area on $\mathcal{D}$. It is easy to see that $Q_{1}=B M O A$, where $B M O A$ is the space of analytic functions of bounded mean oscillation (cf. [5] and [8]). By [1] we know that the spaces $Q_{p}$ are the same and

Received by the editors April 20, 1998 and, in revised form, September 15, 1998.

1991 Mathematics Subject Classification. Primary 30D50.

Key words and phrases. Carleson measure, normal function, the $Q_{p}$ space.

(C)2000 American Mathematical Society 
equal to the Bloch space $\mathcal{B}$ for all $p, 1<p<\infty$. Let $M_{p}$ denote the space of all functions $f$ analytic in $\mathcal{D}$ for which

$$
\sup _{a \in \mathcal{D}} \iint_{\mathcal{D}}\left|f^{\prime}(z)\right|^{2}\left(1-\left|\varphi_{a}(z)\right|^{2}\right)^{p} d A(z)<\infty .
$$

The results in [3] show that $Q_{p}=M_{p}$ for all $p, 0<p<\infty$, or, equivalently, that $f \in Q_{p}$ if and only if $\left|f^{\prime}(z)\right|^{2}\left(1-|z|^{2}\right)^{p} d A(z)$ is a bounded $p$-Carleson measure.

For a meromorphic function $f$, a natural extension of $\left|f^{\prime}(z)\right|$ is the spherical derivative $f^{\#}(z)=\left|f^{\prime}(z)\right| /\left(1+|f(z)|^{2}\right)$, and in the corresponding way to the analytic case we define

$$
Q_{p}^{\#}=\left\{f: f \text { meromorphic in } \mathcal{D} \text { and } \sup _{a \in \mathcal{D}} \iint_{\mathcal{D}}\left(f^{\#}(z)\right)^{2}(g(z, a))^{p} d A(z)<\infty\right\} .
$$

From [1] we know that $Q_{p}^{\#}=N$ for all $p, 1<p<\infty$, where $N$ is the class of normal functions. In the special case $p=1$, the class $Q_{1}^{\#}$ coincides with the class $U B C$ of meromorphic functions of uniformly bounded characteristic in $\mathcal{D}[13$.

Also, for $0<p<\infty$, we consider the following meromorphic analogue of the space $M_{p}$ :

$$
M_{p}^{\#}=\left\{f: f \text { meromorphic in } \mathcal{D} \text { and } \sup _{a \in \mathcal{D}} \iint_{\mathcal{D}}\left(f^{\#}(z)\right)^{2}\left(1-\left|\varphi_{a}(z)\right|^{2}\right)^{p} d A(z)<\infty\right\} .
$$

Notice that the inclusion $Q_{p}^{\#} \subset M_{p}^{\#}$ holds for all $p, 0<p<\infty$. This follows from the inequality $1-\left|\varphi_{a}(z)\right|^{2} \leq 2 g(z, a), z, a \in \mathcal{D}$. A natural idea is, corresponding to the analytic case, to show $Q_{p}^{\#}=M_{p}^{\#}$ for all $p, 0<p<\infty$. Unfortunately, this judgment cannot be proved, and the reason may be complex. However, it is clear that for a meromorphic function $f,\left(f^{\#}(z)\right)^{2}$ may not be subharmonic in general and the expression $1-\left|\varphi_{a}(z)\right|^{2}$, sometimes, cannot be replaced by the Green's function $g(z, a)$ in an integration of $f$ for the meromorphic case.

In this paper we will show that the class $Q_{p}^{\#}$ is not equal to the class $M_{p}^{\#}$ for any $p, 0<p<\infty$, or, equivalently, that for any $p, 0<p<\infty$, there exists a meromorphic function $f \notin Q_{p}^{\#}$ such that $\left(f^{\#}(z)\right)^{2}\left(1-|z|^{2}\right)^{p} d A(z)$ is a bounded $p$ Carleson measure. This corrects Proposition 2 (i) in [4 where "equality" between $Q_{p}^{\#}$ and $M_{p}^{\#}$ was "proved". The proof of Proposition 2 (i) was based on 9, Corollary]. The following theorem shows very strongly that some results for the spaces of analytic functions do not remain true for the corresponding classes of meromorphic functions.

Theorem. There exists an analytic function $f$ such that $f \in \bigcap_{0<p<\infty} M_{p}^{\#}$ but $f \notin \bigcup_{0<p<\infty} Q_{p}^{\#}$.

Before embarking into the proof of the Theorem, let us state a result which has been shown to us in a private communication with Professors O. Reséndiz and L. M. Tovar.

Theorem [11. Let $0<p<\infty$ and let $\left\{z_{n}\right\} \subset \mathcal{D}$ be a sequence. If there exists a constant $M>0$ such that

$$
\sum_{j=k+1}^{\infty}\left(1-\left|z_{j}\right|^{2}\right)^{p} \leq M\left(1-\left|z_{k}\right|^{2}\right)^{p}, \quad k=1,2, \ldots,
$$

then $d \mu(z)=\sum_{n=1}^{\infty}\left(1-\left|z_{n}\right|^{2}\right)^{p} \delta_{z_{n}}$ is a bounded $p$-Carleson measure, where $\delta_{\xi}$ is a Dirac measure at $\xi \in \mathcal{D}$. 
The following theorem is due to Matts Essén and Jie Xiao.

Theorem [7]. Let $B(z)$ be a Blaschke product with zeros in $\left\{z_{n}\right\}, n \in \mathbb{N}$, and $0<$ $p<1$. Then $B(z)$ belongs to the space $Q_{p}$ if and only if $\sum_{n=1}^{\infty}\left(1-\left|z_{n}\right|^{2}\right)^{p} \delta_{z_{n}}$ is a bounded p-Carleson measure.

Proof of the Theorem. Let $0<\beta<1$. We take the sequence $\left\{z_{n}\right\}=\left\{1-\beta^{n}\right\}$ in $\mathcal{D}$ and consider the Blaschke product $B(z)=\prod_{n=1}^{\infty} \frac{z_{n}-z}{1-\bar{z}_{n} z}$ associated with the sequence $\left\{z_{n}\right\}$. By [6] Theorem 9.2] we know that, for $n=1,2, \ldots$,

$$
\prod_{\substack{k=1 \\ k \neq n}}^{\infty}\left|\frac{z_{n}-z_{k}}{1-\bar{z}_{n} z_{k}}\right| \geq \prod_{k=1}^{\infty}\left(\frac{1-\beta^{k}}{1+\beta^{k}}\right)^{2}=\delta>0
$$

Let us consider the function

$$
f(z)=B(z) \log \frac{1}{1-z} \quad(\log 1=0) .
$$

From Lemma 1 in 12 we know that $f \notin N$. Since $Q_{p}^{\#} \subset N$ holds for all $p, 0<p<$ $\infty$, we get $f \notin \bigcup_{0<p<\infty} Q_{p}^{\#}$. Now we turn to prove that $f \in \bigcap_{0<p<\infty} M_{p}^{\#}$. Since $M_{p}^{\#} \subset M_{q}^{\#}$ holds for $0<p<q<\infty$, we need only prove that $f \in \bigcap_{0<p<1} M_{p}^{\#}$. Notice that $|B(z)|<1$ and for each $p, 0<p<1$, we have

$$
\begin{aligned}
\iint_{\mathcal{D}} & \left(f^{\#}(z)\right)^{2}\left(1-\left|\varphi_{a}(z)\right|^{2}\right)^{p} d A(z) \\
\leq & 2 \iint_{\mathcal{D}} \frac{\left|B^{\prime}(z)\right|^{2}|\log (1-z)|^{2}}{\left(1+|f(z)|^{2}\right)^{2}}\left(1-\left|\varphi_{a}(z)\right|^{2}\right)^{p} d A(z) \\
& \quad+2 \iint_{\mathcal{D}}\left|\left(\log \frac{1}{1-z}\right)^{\prime}\right|^{2}\left(1-\left|\varphi_{a}(z)\right|^{2}\right)^{p} d A(z) \\
= & 2\{I(a)+J(a)\} .
\end{aligned}
$$

Since $\log \frac{1}{1-z} \in Q_{p}$ from [2], we get that $\sup _{a \in \mathcal{D}} J(a)<\infty$. Thus we need only prove that $\sup _{a \in \mathcal{D}} I(a)<\infty$.

We take $\delta_{1}=\delta / 4$ and consider all those pseudohyperbolic disks with centers $z_{k}$ and radii $\delta_{1}, k=1,2, \ldots$ It is easy to see that $\mathcal{D}_{k} \cap \mathcal{D}_{n}=\varnothing$, if $k \neq n$, where $\mathcal{D}_{k}=\mathcal{D}\left(z_{k}, \delta_{1}\right), k=1,2, \ldots$. We now estimate the integral $I(a)$ over the regions $E_{1}=\bigcup_{k=1}^{\infty} \mathcal{D}_{k}$ and $E_{2}=\mathcal{D} \backslash E_{1}$. We set $I(a)=I_{1}(a)+I_{2}(a)$, where

$$
I_{i}(a)=\iint_{E_{i}} \frac{\left|B^{\prime}(z)\right|^{2}|\log (1-z)|^{2}}{\left(1+|f(z)|^{2}\right)^{2}}\left(1-\left|\varphi_{a}(z)\right|^{2}\right)^{p} d A(z), \quad i=1,2 .
$$

(i) For any $z \in \partial \mathcal{D}_{k}$, by a simple computation we get

$$
B(z)=-\frac{z-z_{k}}{1-\bar{z}_{k} z} \prod_{\substack{n=1 \\ n \neq k}}^{\infty} \frac{\frac{z_{n}-z_{k}}{1-\bar{z}_{k} z_{n}}-\frac{z-z_{k}}{1-\bar{z}_{k} z}}{1-\overline{\left(\frac{z_{n}-z_{k}}{1-\bar{z}_{k} z_{n}}\right)} \frac{z-z_{k}}{1-\bar{z}_{k} z}}
$$


and then it follows from [8, Lemma 1.4, p. 4 and Lemma 5.2, p. 309] and (1) that

$$
\begin{aligned}
|B(z)| & =\delta_{1} \prod_{\substack{n=1 \\
n \neq k}}^{\infty}\left|\frac{\frac{z_{n}-z_{k}}{1-\bar{z}_{k} z_{n}}-\frac{z-z_{k}}{1-z_{k} z}}{1-\overline{\left(\frac{z_{n}-z_{k}}{1-\bar{z}_{k} z_{n}}\right)} \frac{z-z_{k}}{1-\bar{z}_{k} z}}\right| \geq \delta_{1} \prod_{\substack{n=1 \\
n \neq k}}^{\infty} \frac{\left|\frac{z_{n}-z_{k}}{1-\bar{z}_{k} z_{n}}\right|-\delta_{1}}{1-\delta_{1}\left|\frac{z_{n}-z_{k}}{1-z_{k} z_{n}}\right|} \\
& \geq \delta_{1} \frac{\prod_{\substack{n=1 \\
n \neq k}}^{\infty}\left|\frac{z_{n}-z_{k}}{1-\bar{z}_{k} z_{n}}\right|-\delta_{1}}{1-\delta_{1} \prod_{\substack{n=1 \\
n \neq k}}^{\infty}\left|\frac{z_{n}-z_{k}}{1-\bar{z}_{k} z_{n}}\right|}>\frac{3 \delta^{2}}{16} .
\end{aligned}
$$

Set $B_{m}(z)=\prod_{n=1}^{m} \frac{z_{n}-z}{1-\bar{z}_{n} z}, m \in \mathbb{N}$. Then $B_{m}(z)$ is an analytic function on $\bar{E}_{2}$. It follows from (4) that, for $z \in \partial \mathcal{D}_{k}$,

$$
\left|B_{m}(z)\right| \geq|B(z)|>\frac{3 \delta^{2}}{16} .
$$

We also know that $\left|B_{m}(z)\right|=1$ on $\partial \mathcal{D}$ and $B_{m}(z)$ has no zero points inside $E_{2}$. By the minimum principle and (5) we get

$$
\left|B_{m}(z)\right| \geq \frac{3 \delta^{2}}{16}, \quad z \in E_{2} .
$$

Taking $m \rightarrow \infty$ we obtain that $|B(z)| \geq \frac{3 \delta^{2}}{16}$ for any $z \in E_{2}$. Thus we have

$$
\begin{aligned}
I_{2}(a) & =\iint_{E_{2}} \frac{\left|B^{\prime}(z)\right|^{2}|\log (1-z)|^{2}}{\left(1+|f(z)|^{2}\right)^{2}}\left(1-\left|\varphi_{a}(z)\right|^{2}\right)^{p} d A(z) \\
& \leq \iint_{E_{2}} \frac{\left|B^{\prime}(z)\right|^{2}|\log (1-z)|^{2}}{4|B(z)|^{2}|\log (1-z)|^{2}}\left(1-\left|\varphi_{a}(z)\right|^{2}\right)^{p} d A(z) \\
& \leq \frac{64}{9 \delta^{4}} \iint_{E_{2}}\left|B^{\prime}(z)\right|^{2}\left(1-\left|\varphi_{a}(z)\right|^{2}\right)^{p} d A(z) .
\end{aligned}
$$

Since the zero points of $B(z)$ for $0<p<1$ satisfy

$$
\sum_{j=k+1}^{\infty}\left(1-\left|z_{j}\right|^{2}\right)^{p} \leq \frac{2^{p} \beta^{p}}{1-\beta^{p}}\left(1-\left|z_{k}\right|^{2}\right)^{p}, \quad k=1,2, \ldots,
$$

it follows from Theorem [11] above that $d \mu(z)=\sum_{n=1}^{\infty}\left(1-\left|z_{n}\right|^{2}\right)^{p} \delta_{z_{n}}$ is a bounded $p$-Carleson measure and that $B(z) \in Q_{p}$ by Theorem [7]. Thus, from (6), we have

$$
\sup _{a \in \mathcal{D}} I_{2}(a) \leq \sup _{a \in \mathcal{D}} \frac{64}{9 \delta^{4}} \iint_{\mathcal{D}}\left|B^{\prime}(z)\right|^{2}\left(1-\left|\varphi_{a}(z)\right|^{2}\right)^{p} d A(z)<\infty .
$$

(ii) Now we turn to estimate the integral $I_{1}(a)$. Define

$$
I_{1}^{(k)}(a)=\iint_{\mathcal{D}_{k}} \frac{\left|B^{\prime}(z)\right|^{2}|\log (1-z)|^{2}}{\left(1+|B(z)|^{2}|\log (1-z)|^{2}\right)^{2}}\left(1-\left|\varphi_{a}(z)\right|^{2}\right)^{p} d A(z), \quad k=1,2, \ldots
$$

Let $w=\frac{z-z_{k}}{1-\bar{z}_{k} z}$ and from (3) we have that $B(z)=-w P_{k}(w)$, where

$$
P_{k}(w)=\prod_{\substack{n=1 \\ n \neq k}}^{\infty} \frac{\frac{z_{n}-z_{k}}{1-\bar{z}_{k} z_{n}}-w}{1-\overline{\left(\frac{z_{n}-z_{k}}{1-\bar{z}_{k} z_{n}}\right)} w} .
$$

By (4) and the minimum principle we obtain

$$
\frac{3 \delta}{4} \leq \frac{\delta-\delta_{1}}{1-\delta \delta_{1}} \leq\left|P_{k}(w)\right|<1, \quad|w|<\delta_{1} .
$$


Since $z_{k}, k=1,2, \ldots$, are real numbers, we have

$$
1-z=\left(1-z_{k}\right) \frac{1-w}{1+\bar{z}_{k} w}
$$

Using the inequalities

$$
\frac{1-\delta}{1+\delta}<\left|\frac{1-w}{1+\bar{z}_{k} w}\right|<\frac{1+\delta}{1-\delta}
$$

for $|w|<\delta_{1}$ and, by (10), we get that there exist positive numbers $c_{1}=c_{1}(\delta), c_{2}=$ $c_{2}(\delta)$ and $c(\delta)$ such that

$$
\left|\log c_{1}\left(1-z_{k}\right)\right| \leq|\log (1-z(w))| \leq\left|\log c_{2}\left(1-z_{k}\right)\right|, \quad|w|<\delta_{1},
$$

and

$$
\left|\frac{\log c_{2}\left(1-z_{k}\right)}{\log c_{1}\left(1-z_{k}\right)}\right| \leq c(\delta), \quad k=1,2, \ldots
$$

where the constant $c(\delta)$ depends only on $\delta$. A computation shows that

$$
\begin{aligned}
\left(1-\left|\varphi_{a}(z)\right|^{2}\right)^{p} & =\left(1-\left|\frac{z_{k}-a}{1-\bar{a} z_{k}}\right|^{2}\right)^{p} \frac{\left(1-|w|^{2}\right)^{p}}{\left|1+\overline{\left(\frac{z_{k}-a}{1-a z_{k}}\right)} w\right|^{2 p}} \\
& \leq(5 / 3)\left(1-\left|\frac{z_{k}-a}{1-\bar{a} z_{k}}\right|^{2}\right)^{p} \\
& =C\left(z_{k}, a, p\right),
\end{aligned}
$$

where $w=\frac{z-z_{k}}{1-\overline{z_{k}} z}$ and $|w|<\delta_{1}$. From $B^{\prime}(z)=-w^{\prime}(z)\left(P_{k}(w)+w P_{k}^{\prime}(w)\right)$ we get

$$
\left|B^{\prime}(z)\right|^{2} \leq 2\left|w^{\prime}(z)\right|^{2}\left(\left|P_{k}(w)\right|^{2}+|w|^{2}\left|P_{k}^{\prime}(w)\right|^{2}\right)
$$

which, with (8) and (13), gives

$$
\begin{aligned}
I_{1}^{(k)}(a)= & \iint_{\mathcal{D}_{k}} \frac{\left|B^{\prime}(z) \log (1-z)\right|^{2}}{\left(1+|B(z) \log (1-z)|^{2}\right)^{2}}\left(1-\left|\varphi_{a}(z)\right|^{2}\right)^{p} d A(z) \\
\leq & C\left(z_{k}, a, p\right) \iint_{\mathcal{D}_{k}} \frac{\left(\left|P_{k}(w)\right|^{2}+\left|w P_{k}^{\prime}(w)\right|^{2}\right)|\log (1-z)|^{2}}{\left(1+\left|w P_{k}(w) \log (1-z)\right|^{2}\right)^{2}}\left|w^{\prime}(z)\right|^{2} d A(z) \\
\leq & C\left(z_{k}, a, p\right)\left\{\iint_{|w|<\delta_{1}} \frac{|\log (1-z(w))|^{2}}{\left(1+\left|w P_{k}(w) \log (1-z(w))\right|^{2}\right)^{2}} d A(w)\right\} \\
& \quad+C\left(z_{k}, a, p\right)\left\{\iint_{|w|<\delta_{1}} \frac{\left|w P_{k}^{\prime}(w) \log (1-z(w))\right|^{2}}{\left(1+\left|w P_{k}(w) \log (1-z(w))\right|^{2}\right)^{2}} d A(w)\right\} \\
= & C\left(z_{k}, a, p\right)\left\{I_{11}^{(k)}+I_{12}^{(k)}\right\} .
\end{aligned}
$$


By (9), (11) and (12) we obtain

$$
\begin{aligned}
I_{11}^{(k)} & =\iint_{|w|<\delta_{1}} \frac{|\log (1-z(w))|^{2}}{\left(1+\left|w P_{k}(w) \log (1-z(w))\right|^{2}\right)^{2}} d A(w) \\
& \leq \iint_{|w|<\delta_{1}} \frac{\left|\log c_{2}\left(1-z_{k}\right)\right|^{2}}{\left(1+\frac{9}{16} \delta^{2}|w|^{2}\left|\log c_{1}\left(1-z_{k}\right)\right|^{2}\right)^{2}} d A(w) \\
& \leq 2 \pi \int_{0}^{\delta_{1}} \frac{\left|\log c_{2}\left(1-z_{k}\right)\right|^{2}}{\left(1+\frac{9}{16} \delta^{2}\left|\log c_{1}\left(1-z_{k}\right)\right|^{2} t^{2}\right)^{2}} t d t \\
& \leq \frac{16 \pi}{9 \delta^{2}}(c(\delta))^{2}
\end{aligned}
$$

Since $P_{k}(w)$ is continuous in $|w| \leq \delta_{1}$, from (9) we have

$$
\begin{aligned}
I_{12}^{(k)} & =\iint_{|w|<\delta_{1}} \frac{\left|w P_{k}^{\prime}(w) \log (1-z(w))\right|^{2}}{\left(1+\left|w P_{k}(w) \log (1-z(w))\right|^{2}\right)^{2}} d A(w) \\
& \leq \frac{4}{9 \delta^{2}} \iint_{|w|<\delta_{1}}\left|P_{k}^{\prime}(w)\right|^{2} d A(w) \\
& \leq \frac{1}{2 \delta^{2}} \iint_{\mathcal{D}}\left|P_{k}^{\prime}(w)\right|^{2}\left(1-|w|^{2}\right) d A(w) \\
& \leq b(\delta)\left\|P_{k}\right\|_{H^{2}}^{2},
\end{aligned}
$$

where $b(\delta)$ is a constant depending only on $\delta$ and

$$
\left\|P_{k}\right\|_{H^{2}}^{2}=\sup _{0<r<1} \frac{1}{2 \pi} \int_{0}^{2 \pi}\left|P_{k}\left(r e^{i \theta}\right)\right|^{2} d \theta \leq 1 .
$$

Thus, from (14), (15) and (16), we have

$$
\begin{aligned}
I_{1}^{(k)}(a) & \leq(10 / 3)\left(1-\left|\frac{z_{k}-a}{1-\bar{a} z_{k}}\right|^{2}\right)^{p}\left\{I_{11}^{(k)}+I_{12}^{(k)}\right\} \\
& \leq C(\delta)\left(1-\left|\frac{z_{k}-a}{1-\bar{a} z_{k}}\right|^{2}\right)^{p},
\end{aligned}
$$

where the constant $C(\delta)$ depends only on $\delta$.

Since $d \mu(z)=\sum_{n=1}^{\infty}\left(1-\left|z_{n}\right|^{2}\right)^{p} \delta_{z_{n}}$ is a bounded $p$-Carleson measure in $\mathcal{D}$, from (8), (17) and Lemma 2.1 in [3] we have

$$
\sup _{a \in \mathcal{D}} I_{1}(a)=\sup _{a \in \mathcal{D}} \sum_{k=1}^{\infty} I_{1}^{(k)}(a) \leq C(\delta) \sup _{a \in \mathcal{D}} \sum_{k=1}^{\infty}\left(1-\left|\frac{z_{k}-a}{1-\bar{a} z_{k}}\right|^{2}\right)^{p}<\infty .
$$

Combining (2), (7) and (18) we get

$$
\sup _{a \in \mathcal{D}} \iint_{\mathcal{D}}\left(f^{\#}(z)\right)^{2}\left(1-\left|\varphi_{a}(z)\right|^{2}\right)^{p} d A(z)<\infty
$$

that is, $f \in M_{p}^{\#}$. Thus $f \in \bigcap_{0<p<\infty} M_{p}^{\#}$. This completes the proof of the Theorem.

Corollary 1. $Q_{p}^{\#} \varsubsetneqq M_{p}^{\#}$ for any $p, 0<p<\infty$.

Corollary 2. There exists a non-normal function $f$ such that

$$
\left(f^{\#}(z)\right)^{2}\left(1-|z|^{2}\right) d A(z)
$$

is a Carleson measure. 
Remark 1. The theorem above shows that the class $Q_{p}^{\#}$ cannot be characterized by a general bounded $p$-Carleson measure for any $p, 0<p<\infty$, and this is an obvious difference between the analytic and meromorphic case. It is necessary to point out that our proof uses some ideas employed in [10].

Remark 2. Recently, the classes $Q_{p}^{\#}$ have been characterized by other Carleson measures that are called Carleson type measures, which were introduced by the second author in his paper [14. Also, some further relations between the classes $Q_{p}^{\#}$ and $M_{p}^{\#}$ are described there; one of them is that $Q_{p}^{\#}=N \cap M_{p}^{\#}$ for all $p$, $0<p<\infty$.

\section{ACKNOWLEDGEMENT}

We would like to thank the referee for several helpful suggestions.

\section{REFERENCES}

[1] R. Aulaskari and P. Lappan, Criteria for an analytic function to be Bloch and a harmonic or meromorphic function to be normal, Complex analysis and its applications, Pitman Research Notes in Mathematics, 305, Longman Scientific \& Technical, Harlow, 1994, pp. 136-146. MR 95g:30045

[2] R. Aulaskari, P. Lappan, J. Xiao and R. Zhao, On $\alpha$-Bloch spaces and multipliers of Dirichlet spaces, J. Math. Anal. Appl. 209 (1997), 103-121. MR 98e:30036

[3] R. Aulaskari, D. A. Stegenga and J. Xiao, Some subclasses of BMOA and their characterization in terms of Carleson measure, Rocky Mountain J. Math. 26 (1996), 485-506. MR 97k:30045

[4] R. Aulaskari, J. Xiao and R. Zhao, On subspaces and subsets of BMOA and UBC, Analysis 15 (1995), 101-121. MR 96j:30050

[5] A. Baernstein II, Analytic functions of bounded mean oscillation, Aspects of contemporary complex analysis, Academic Press, London, 1980, pp. 3-36. MR 84g:42021

[6] P. L. Duren, Theory of $H^{p}$ spaces, Academic Press, New York, 1970. MR 42:3552

[7] M. Essén and J. Xiao, Some results on $Q_{p}$ spaces, $0<p<1$, J. Reine Angew. Math. 485 (1997), 173-195. MR 98d:46024

[8] J. B. Garnett, Bounded analytic functions, Academic Press, New York, 1981. MR 83g:30037

[9] Z̆. Pavićević, The Carleson measure and meromorphic functions of uniformly bounded characteristic, Ann. Acad. Sci. Fenn. Ser. A I Math. 16 (1991), 249-254. MR 93h:30044

[10] J. Rong and J. Huang, The Carleson measure characteristics of UBC functions, J. Math. (Wuhan) 10 (1990), 255-262. MR 92f:30051

[11] O. Reséndiz and L. M. Tovar, Carleson measures, Blaschke products and $Q_{p}$ spaces, manuscript.

[12] S. Yamashita, A non-normal function whose derivative has finite area integral of order $0<p<2$, Ann. Acad. Sci. Fenn. Ser. A I Math. 4 (1978/1979), 293-298. MR 81k:30042

[13] S. Yamashita, Functions of uniformly bounded characteristic, Ann. Acad. Sci. Fenn. Ser. A I Math. 7 (1982), 349-367. MR 84d:30060

[14] H. Wulan, On some classes of meromorphic functions, Ann. Acad. Sci. Fenn. Math. Diss. 116 (1998), 1-57. CMP 99:01

Department of Mathematics, University of Joensud, P.O. Box 111, Fin-80101 Joensuu, FINLAND

E-mail address: Rauno.Aulaskari@joensuu.fi

E-mail address: wulan@cc.joensuu.fi

Department of Mathematics, Inner Mongolia Normal University, Hohhot 010022, People's Republic of China

Department of Mathematics, Faculty of Science, Kyoto University, Kyoto 606-8502, JAPAN

E-mail address: zhao@kusm.kyoto-u.ac.jp 